\title{
Immunodeficiency-Like Phenotype, Recurrent Pulmonary Manifestations, and Persistent Polyarthritis: Mevalonate Kinase Deficiency Successfully Treated With Adalimumab
}

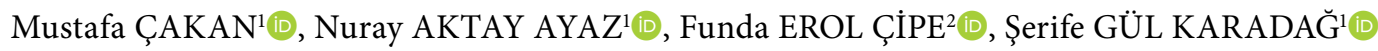 \\ ${ }^{1}$ Department of Child Health and Diseases, Division of Pediatric Rheumatology, Kanuni Sultan Süleyman \\ Research and Training Hospital, İstanbul, Turkey \\ ${ }^{2}$ Department of Pediatric Allergy and Immunology, Kanuni Sultan Süleyman Research and Training Hospital, İstanbul, Turkey
}

\begin{abstract}
Mevalonate kinase deficiency (MKD) is an autosomal recessive autoinflammatory disorder characterized by fever starting in early infancy, lasting for three to seven days, recurring every one to two months, and accompanied by diarrhea, rash, abdominal pain, oral aphthous ulcers, transient arthritis and cervical lymphadenopathy. ${ }^{1-4}$ There are reports of MKD patients with severe gastrointestinal involvement mimicking inflammatory bowel disease, interstitial lung disease, and with recurrent bacterial infections mimicking more like an immunodeficiency phenotype. ${ }^{1,2,5}$ Anti-interleukin 1 treatment seems to be the most efficacious treatment modality but there are few reports on the beneficial effect of anti-tumor necrosis factor. ${ }^{7,8}$
\end{abstract}

A 15-year-old girl was admitted to pediatric rheumatology out-patient clinic with the complaint of joint swellings for the last two months. She had arthritis on bilateral ankles, knees and elbows. Medical history was remarkable for periodic fever attacks that started around four years of age, lasting 7-10 days, recurring every three-four months. Around five years of age, she started to have recurrent wheezing and pneumonia attacks and was hospitalized 11 times mostly because of the respiratory problems. She had diagnosis of asthma and bronchiectasis at the age of seven and inhaler treatments were started. She was having at least three-four attacks of fever, some with wheezing every year. She was investigated for immunodeficiency, tuberculosis and cystic fibrosis because of the recurrent lung infections and bronchiectasis. All tests including tuberculosis screen tests, allergy tests, bronchoscopic examination, serum immunoglobulins, lymphocyte subtypes, sweat chloride test, and nitroblue tetrazolium test were negative. At the age of 12 , she started to have recurrent joint swellings with fever on the ankles and knees resolving in two-three weeks without any abdominal or chest pain. Familial Mediterranean fever was considered in the differential diagnosis and colchicine trial was performed (Mediterranean fever gene analysis: no mutation) but was ineffective on fever and arthritis attacks. At the age of 15 , she was referred to pediatric rheumatology due to persistent arthritis, diagnosed as rheumatoid factor negative polyarticular juvenile idiopathic arthritis, and started methotrexate $\left(15 \mathrm{mg} / \mathrm{m}^{2} /\right.$ weekly,

\footnotetext{
Received: September 08, 2019 Accepted: October 13, 2019 Published online: January 08, 2020

Correspondence: Mustafa Çakan, MD. Kanuni Sultan Süleyman Eğitim ve Araştırma Hastanesi, Çocuk Sağlığı ve Hastalıkları Kliniği, Pediatrik Romatoloji Bölümü, 34303 Küçükçekmece, İstanbul, Türkiye. Tel: +90 212 - 4041500 e-mail: mustafacakan@hotmail.com Mevalonate Kinase Deficiency Successfully Treated With Adalimumab. Arch Rheumatol 2020;35(4):627-628. 
subcutaneous). After three months, arthritis did not improve and adalimumab was added (40 mg/subcutaneous/every two weeks). At this time, whole exome sequencing was performed and it was seen that she had compound heterozygous mutation (G18R/V377I) in the mevalonate kinase gene. The mutation was confirmed with Sanger sequencing. The child was diagnosed as having MKD 12 years after the first symptom. She has been using adalimumab for 36 months and we have observed a dramatic response with resolution of arthritis, fever attacks and pulmonary symptoms since the first dose of adalimumab.

In conclusion, in children presenting with an immunodeficiency-like phenotype and recurrent pulmonary symptoms that could not be explained by an extensive work-up, MKD should be considered in the differential diagnosis. This case broadens the clinical spectrum of MKD and also emphasizes the importance of international collaborations and whole exome sequencing in patients with complex phenotype. To the best of our knowledge, this is one of the first reports on the efficacy of adalimumab in MKD.

Informed consent was received from the legal guardians of the child.

\section{Acknowledgements}

The genetic study was performed in the laboratory of Dr. R. Geha and J. Chou in the Division of Immunology, Children's Hospital, Harvard Medical School, Boston, USA.

\section{Declaration of conflicting interests}

The authors declared no conflicts of interest with respect to the authorship and/or publication of this article.

\section{Funding}

The authors received no financial support for the research and/or authorship of this article.

\section{REFERENCES}

1. Ter Haar NM, Jeyaratnam J, Lachmann HJ, Simon A, Brogan PA, Doglio M, et al. The phenotype and genotype of mevalonate kinase deficiency: a series of 114 cases from the eurofever registry. Arthritis Rheumatol 2016;68:2795-805.

2. Bader-Meunier B, Florkin B, Sibilia J, Acquaviva C, Hachulla E, Grateau G, et al. Mevalonate kinase deficiency: a survey of 50 patients. Pediatrics 2011;128:e152-9.

3. Şen H, Sılan F, Binnetoğlu E, Güneş F, Akurut Ç, Uludağ A, et al. Hyperimmunoglobulin D syndrome: Case report. Arch Rheumatol 2015;30:244-6.

4. Gattorno M, Hofer M, Federici S, Vanoni F, Bovis F, Aksentijevich I, et al. Classification criteria for autoinflammatory recurrent fevers. Ann Rheum Dis 2019;78:1025-32.

5. Pietrasanta C, Minoia F, Torreggiani S, Ronchi A, Gattorno M, Volpi S, et al. When neonatal inflammation does not mean infection: an early-onset mevalonate kinase deficiency with interstitial lung disease. Clin Immunol 2019;205:25-8.

6. De Benedetti F, Gattorno M, Anton J, Ben-Chetrit E, Frenkel J, Hoffman HM, et al. Canakinumab for the treatment of autoinflammatory recurrent fever syndromes. N Engl J Med 2018;378:1908-19.

7. Demirkaya E, Caglar MK, Waterham HR, Topaloglu $\mathrm{R}$, Ozen S. A patient with hyper-IgD syndrome responding to anti-TNF treatment. Clin Rheumatol 2007;26:1757-9.

8. Di Gangi M, Amato G, Converso G, Benenati A, Leonetti C, Borella E, et al. Long-term efficacy of adalimumab in hyperimmunoglobulin $D$ and periodic fever syndrome. Isr Med Assoc J 2014;16:605-7. 